\title{
OCCLUSION OF THE CENTRAL RETINAL ARTERY AND OCULAR NEOVASCULARISATION: AN INDIRECT ASSOCIATION?
}

\author{
N. A. JACOBS and D. R. TREW \\ London and Kingston
}

\begin{abstract}
SUMMARY
Ocular neovascularisation is recognised as an uncommon complication of central retinal artery occlusion (CRAO) but has been associated most frequently in those patients suffering from carotid artery occlusive disease. We report the finding of neovascularisation after CRAO in a group of 8 patients, who have presented over the previous 6 years. Four showed no evidence of atherosclerotic carotid disease and only 2 were diabetic. Although the group numbers are necessarily small, it is suggested that these cases support the assertion that ocular neovascularisation may occur following CRAO in the absence of any underlying and predisposing ischaemic or inflammatory condition.
\end{abstract}

The relationship between ocular neovascularisation and central retinal artery occlusion (CRAO) merits clarification. Their association was first described by Loring ${ }^{\prime}$ and more recently reviewed by Hayreh and Podhajsky. ${ }^{2}$ Duker and Brown $^{3}$ found that new vessel disease occurred in $1.8 \%$ of cases of CRAO. Of the 8 patients described in this paper who developed ocular neovascularisation, only 4 had demonstrable carotid artery disease and only 2 were diabetic. The relationship between any underlying disease, the arterial occlusion and neovascularisation is discussed. Carotid angiography and fundal photography from the first case are included for illustration.

\section{CASE REPORTS}

\section{Case 1}

A 60-year-old man gave a history of intermittent left arm weakness for a year, right amaurosis fugax for 2 months and one episode of horizontal diplopia shortly before presenting with a complete right IIIrd nerve palsy. A loud

From: Department of Ophthalmology, Charing Cross Hospital, London W6, and Royal Eye Unit, Kingston Hospital, Surrey, UK.

Correspondence to: Mr. D. R. Trew, Department of Ophthalmology, Charing Cross Hospital, London W6 8RF, UK. bruit was heard over the right carotid artery and angiography revealed internal carotid artery occlusion with long concentric external carotid stenosis on that side (Fig. 1). General examination and investigation was otherwise completely normal.

One month after initial presentation he suffered a right CRAO and 7 weeks later returned complaining of an ache over this eye. On examination he was found to have episcleral injection, corneal oedema and rubeosis iridis. The

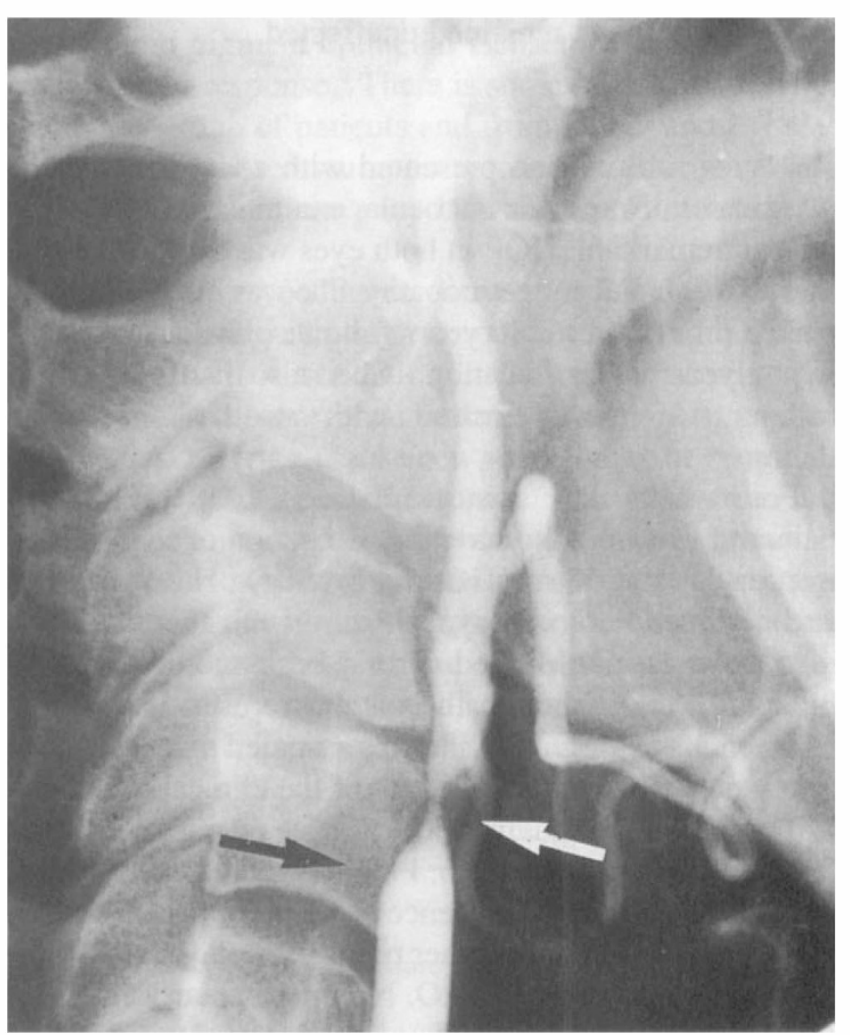

Fig. 1. Right carotid angiogram from case I, showing internal carotid occlusion (black arrow) with external carotid stenosis (white arrow). 


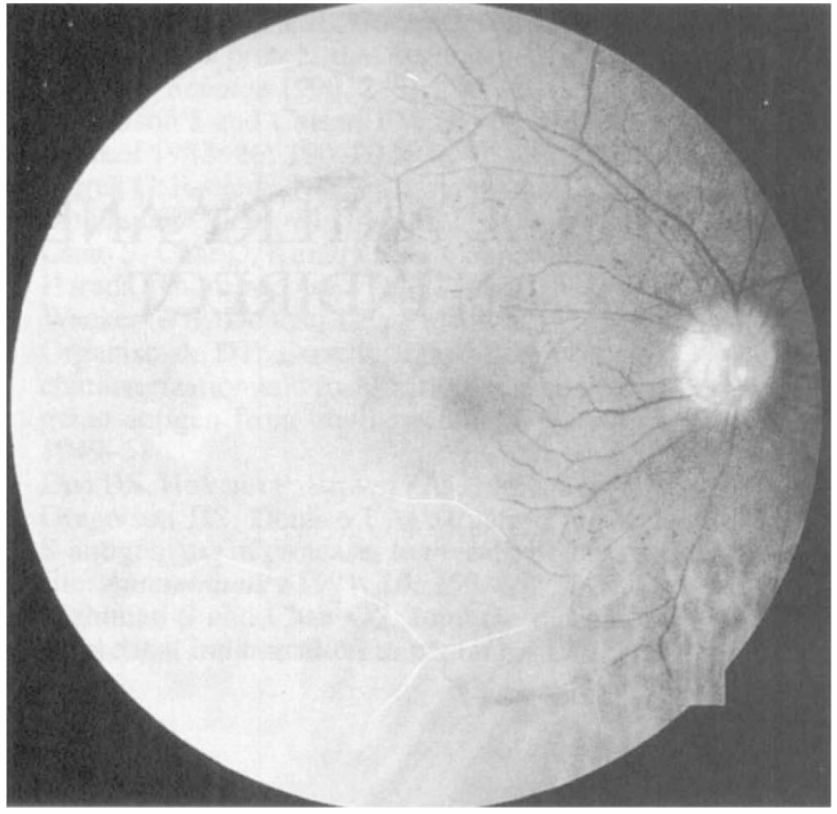

Fig. 2. Right red-free photograph from case 1, showing established central retinal artery occlusion and disc new vessels.

intraocular pressure (IOP) was $25 \mathrm{mmHg}$. Disc new vessels (Fig. 2) were noted after a further 6 weeks, with increased rubeosis and an IOP of $35 \mathrm{mmHg}$.

Seven months after initial presentation the IIIrd nerve palsy had resolved. Right visual acuity was no perception of light with a vitreous haemorrhage obscuring the fundal view. The left eye remained unaffected.

\section{Case 2}

A 71-year-old woman presented with a left CRAO. She was bilaterally aphakic but ocular examination was otherwise unremarkable, IOP in both eyes was $10 \mathrm{mmHG}$ and there was no diabetic retinopathy. She was a diabetic, controlled on insulin for 10 years following failure with oral hypoglycaemic medication. She also suffered from asthma which was treated with standard inhalation therapy.

Four weeks later she complained of left peri-orbital pain and was noted to have no perception of light in that eye, an IOP of $52 \mathrm{mmHG}$ and extensive rubeosis iridis and peripheral anterior synechiae, though there were no disc new vessels. Marked carotid bruits could be heard bilaterally and although the patient refused to undergo intravenous angiography she was assumed to have carotid artery occlusive disease in view of the clinical findings.

The patient eventually underwent an enucleation of the left eye for intractable pain. Pathological examination of the eye confirmed the presence of iris new vessels and an almost complete loss of inner retinal fibre layers and ganglion cells due to the CRAO. No micro-aneurysms were identified in any section.

\section{Case 3}

A 61-year-old man presented giving a history of complete visual loss in the right eye 2 weeks previously. In the 3 months before this event he had suffered nearly 20 episodes of transient visual loss lasting 15-30 minutes on each occasion and his vision had been 'little more than a blur' during this period. He was a controlled systemic hypertensive and suffered from angina and fibrosing alveolitis.

On examination the right eye had no perception of light and a relative afferent pupil defect. There was rubeosis iridis, and also an anterior uveitis with posterior synechiae. New vessels were present at the disc and cholesterol plaques throughout the fundus. The IOP was $6 \mathrm{mmHg}$. The left eye showed thinned retinal arterioles but was otherwise normal. Ophthalmodynamometry revealed diastolic values of $5 \mathrm{mmHg}$ for the right eye and $22 \mathrm{mmHg}$ for the left. He was diagnosed as having suffered a right CRAO in an eye with chronic ocular ischaemia. Carotid angiography demonstrated a severe stenosis of the right internal carotid artery with complete occlusion of the origin of the right external carotid.

\section{Case 4}

A 70-year-old West Indian man presented with visual loss in the left eye and the typical fundal appearances of a CRAO with a pale retina, cherry red spot and attenuated arterioles. This had occurred suddenly and left him with perception of light only in that eye. Six weeks later he developed ocular pain and on examination was found to have chemosis, corneal oedema, rubeosis iridis and an IOP of $68 \mathrm{mmHg}$. He was a diabetic, diagnosed 2 years previously, and treated with oral hypoglycaemic medication. Angiography of his carotid arteries failed to show any gross abnormality.

A year later the patient suffered a right upper temporal branch artery occlusion. Neither rubeosis iridis nor disc new vessels developed and a further angiogram again showed a fully patent carotid system.

\section{Case 5}

A 78-year-old man presented with sudden, painless visual loss in the left eye and the fundal appearances of a CRAO. The visual acuity was 'hand movements' only. Nine weeks later he was found to have suffered a vitreous haemorrhage and when this cleared, new vessels were seen to be present at the disc. He underwent panretinal photocoagulation and the new vessels regressed; rubeosis iridis did not develop and the visual acuity remained at 'hand movements'.

His carotid arteries were fully patent but he had suffered a myocardial infarction 10 years earlier and was in controlled atrial fibrillation. He had made a good recovery from a previous right cerebrovascular accident and a prostatectomy had been performed for carcinoma some years before. There was no evidence of any metastatic spread. He was treated also for gout.

\section{Case 6}

A 67-year-old man presented with loss of vision in the right eye and was diagnosed as having a CRAO. He had 
Table I. Relevant clinical findings in a group of 8 patients who developed ocular neovascularisation following central retinal artery obstruction

\begin{tabular}{|c|c|c|c|c|c|c|c|c|}
\hline Age & Sex & Eye & NVD & NVI & Carotid & Diabetes & Other & Outcome \\
\hline 60 & $\mathbf{M}$ & $\mathrm{R}$ & + & + & + & - & - & NPL \\
\hline 71 & $\mathrm{~F}$ & $\mathrm{~L}$ & - & + & + & + & Asthma & Enucleation \\
\hline 61 & $\mathbf{M}$ & $\mathrm{R}$ & + & + & + & - & $\mathrm{BP} \uparrow+\mathrm{OI}$ & NPL \\
\hline 70 & $\mathbf{M}$ & $\mathrm{L}$ & - & + & - & + & - & PL \\
\hline 78 & $\mathbf{M}$ & $\mathrm{L}$ & + & - & - & - & $\mathrm{MI}+\mathrm{AF}$ & HM \\
\hline 67 & $\mathbf{M}$ & $\mathrm{R}$ & - & + & + & - & - & PL \\
\hline 88 & $\mathrm{~F}$ & $\mathrm{~L}$ & - & + & - & - & - & PL \\
\hline 75 & $\mathrm{~F}$ & $\mathrm{R}$ & - & + & - & - & $\mathrm{BP} \uparrow$ & NPL \\
\hline
\end{tabular}

Key: NVD, new vessels at disc; NVI, new vessels on iris (rubeosis); $\mathrm{BP} \uparrow$, systemic hypertensive; OI, chronic ocular ischaemia; MI, myocardial infarction; AF, atrial fibrillation; (N)PL: (no) perception of light; HM, hand movements only.

suffered episodes of transient visual loss during the previous few months but had made a full recovery on these occasions after 20-30 minutes. Within 10 weeks he had developed rubeosis iridis and examination revealed anterior chamber flare and an IOP of $37 \mathrm{mmHg}$. There were no disc new vessels. Carotid angiography demonstrated a complete occlusion of the right internal carotid artery at its origin. Five months after presentation the right lens began to opacify and soon formed a dense cataract. The left eye remained unaffected.

His general history appeared unremarkable and on examination and investigation there was no evidence of diabetes mellitus, systemic hypertension or other predisposing ischaemic condition.

\section{Case 7}

An 88-year-old woman presented with a CRAO in her aphakic left eye. IOP was $16 \mathrm{mmHg}$. Three months later she returned complaining of soreness and redness of this eye. Visual acuity was 'perception of light', there was corneal oedema, a dilated pupil and the IOP was $66 \mathrm{mmHg}$. Marked rubeosis iridis was present. Panretinal laser ablation was performed but fine rubeotic vessels persisted with extensive peripheral anterior synechiae formation. The phakic right eye remained healthy throughout.

On general examination, no clinical or laboratory evidence was found of any predisposing ischaemic condition. Intravenous digital subtraction angiography was of good quality and failed to reveal any stenotic disease of the carotid vessels.

\section{Case 8}

A 75-year-old woman presented with a CRAO in the right eye. She was hypertensive $(190 / 120 \mathrm{mmHg})$ and was therefore started on oral atenolol which successfully controlled her blood pressure. Other investigations were normal but she was also commenced on $75 \mathrm{mg}$ aspirin daily.

Twelve weeks later she presented to her general practitioner in considerable pain from her right eye. Examination demonstrated marked rubeosis of the iris and angle and an IOP of $65 \mathrm{mmHg}$. Visual acuity was 'no perception of light' and new blood vessels were not seen at the optic disc or elsewhere in the retina. Again, haematological and biochemical investigations were normal. Echocardiography was unremarkable and carotid Doppler ultrasound examination failed to show any evidence of atheromatous narrowing; blood pressure was $160 / 90 \mathrm{mmHg}$. Pain in her blind right eye was relieved by the use of topical steroids and mydriatics.

\section{DISCUSSION}

Table I presents the relevant clinical features of our group of 8 patients and shows that only 4 demonstrated carotid artery occlusive disease. One was also diabetic but neither she nor the other diabetic patient demonstrated retinopathy before the onset of CRAO-related ocular neovascularisation. One of the 'non-carotid disease' patients had previously suffered a myocardial infarction and cerebrovascular accident and presented in controlled atrial fibrillation, whilst another was hypertensive. There were no other predisposing inflammatory or ischaemic conditions discovered on examination and investigation of these patients.

Totally anoxic retinal tissue is deemed unable to provide the necessary stimulus for neovascularisation, ${ }^{4}$ so that the distinction between ischaemia and necrosis is critical. The origin of the neovascular stimulus following CRAO is unclear but may therefore be dependent upon a further ischaemic insult to the eye, such as the presence of carotid artery occlusive disease or a posterior ciliary artery occlusion. A combination of pathology could lead to outer retinal and pigment epithelial ischaemia ${ }^{5}$ and result in a neovascular response. ${ }^{6}$ There is some evidence, however, from our group of patients and from Duker and Brown's review $^{3}$ that these assumptions may not always be valid and that CRAO alone may be capable of initiating this response.

Carotid artery occlusive disease giving rise to ocular neovascularisation through chronic ischaemia of the eye has been well described. ${ }^{5-9}$ Central nervous system involvement is more usual in these patients than reported with CRAO ${ }^{10}$ Iris and disc new vessels may be seen associated with other signs of chronic ocular ischaemia, such as anterior segment inflammation ${ }^{7}$ and venous stasis retinopathy. ${ }^{11}$ However, the ensuing rubeotic glaucoma is often, though not invariably, characterised by a relatively low IOP due to decreased ciliary body perfusion as a result of carotid artery occlusive disease. ${ }^{5,7,12}$ Nevertheless, it appears that even this reduced IOP may be associated with

Table II. A classification of the proposed mechanisms relating central retinal artery occlusion (CRAO) to ocular neovascularisation (NVN)
1. Chronic ocular ischaemia alone leading to $\rightarrow$ rubeotic ocular NVN
Low CRA perfusion pressure glaucoma ${ }^{\mathrm{a}}$ $\rightarrow \mathrm{CRAO}$
2. Chronic ocular ischaemia + coincidental CRAO $\rightarrow$ ocular NVN
3. Double embolism: CRAO + PCAO $^{\mathrm{b}} \rightarrow$ ocular NVN
4. CRAO alone $\rightarrow$ ocular NVN

${ }^{\text {a}}$ Rubeotic glaucoma is usually characterised by a low intraocular pressure.

${ }^{6}$ PCAO, posterior ciliary artery occlusion. 
occlusion of the central retinal artery, whose own perfusion pressure is reduced by the same underlying pathology. ${ }^{2.5}$ This possible association between ocular neovascularisation and CRAO is illustrated in Table II. Hayreh and Podhajsky stated that it accounted for the majority of such cases ${ }^{2}$ and Brown et al. considered that most of their comparable patients also fell into this category. ${ }^{5}$

CRAO may also arise as a separate embolic manifestation of carotid occlusive disease in the setting of chronic ocular ischaemia. ${ }^{2}$ It would seem, therefore, that CRAO may indeed precede neovascularisation. Case 1, whose proven occlusive disease is illustrated in Fig. 1, must fall into one of the two categories described above (categories 1 and 2 in Table II). Inflammatory anterior segment changes and rubeotic glaucoma were not noted until 7 weeks after CRAO. The second case, with a low IOP initially, is more likely to belong to the second category. Kearns et al. have described a similar case. ${ }^{12}$

A further possible cause of ocular ischaemia could be occlusion of the posterior ciliary artery system. Perraut and Zimmerman, in their pathological study, described 6 cases presenting with rubeotic glaucoma within 9 weeks of CRAO. ${ }^{13}$ Wolter and Phillips have also reported the pathology of 3 further cases, including a diagnosis of temporal arteritis. ${ }^{14-16}$ They described the phenomenon of 'double embolism', i.e. occlusion of both central retinal and any number of posterior ciliary arteries (category 3 in Table II). These patients demonstrated high IOP. Hayreh and Podhajsky felt that the case for double embolism was not proved, pointing out that the existence of carotid occlusive disease was largely ignored in these earlier reports. Furthermore, clinical evidence of double embolism would be difficult to obtain, requiring both the exclusion of carotid disease and the demonstration of a choroidal circulatory deficit at the onset of neovascularisation, although fluorescein angiography may be sufficient for the latter purpose.

Finally, there is the possibility that CRAO alone may be responsible for neovascular changes. Four of our patients did not demonstrate carotid artery occlusive disease and one of these had no evident predisposing cardiovascular pathology at all. Kottow and Hendrickson suggested that anterior segment neovascularisation may follow certain observed alterations in the iris circulation after CRAO. ${ }^{17}$ Brown recently reported a case following CRAO due to a mitral valve embolus in the absence of carotid disease. ${ }^{18}$ He proposed that a chronic state of inner retinal hypoxia may have developed. Although the choroidal circulation was normal at the time of the CRAO, the chance of a posterior ciliary artery occlusion developing subsequently, and before the appearance of new vessels, cannot be excluded.

The retrospective nature of most reports concerning the association described in this paper often makes it impossible to be certain of the sequence of events. This is primarily because CRAO is thought of as a 'retinal' diagnosis, making a patient with this complaint unlikely to receive a thorough anterior segment examination in the first instance. However, we have shown that ocular neovascularisation may occur following CRAO even in the absence of obvious cardiovascular signs. In view of the progression demonstrated by this group of patients, we would recommend that patients who have suffered CRAO should be followed for approximately 3 months with regard to the possible development of neovascular disease and for appropriate treatment.

Key words: Central retinal artery, Neovascularisation.

\section{REFERENCES}

1. Loring EG: Remarks on embolism. Am J Med Sci 1874, 67: 313-28.

2. Hayreh SS and Podhajsky P: Ocular neovascularisation with retinal vascular occlusion. II: Occurrence in central and branch retinal artery occlusion. Arch Ophthalmol 1982, 100: $1585-96$.

3. Duker JS and Brown GC: Neovascularisation of the optic disc associated with obstruction of the central retinal artery. Ophthalmology 1989; 96: 87-91.

4. Henkind $P$ and Wise GN: Retinal neovascularisation, collaterals and vascular shunts. $\mathrm{Br} J$ Ophthalmol 1974, 58: 413-22.

5. Brown GC, Magargal LE, Simeone FA, Goldberg RE, Federman JL, Benson WE: Arterial obstruction and ocular neovascularisation. Ophthalmology 1982, 89: 132-46.

6. Federman JL, Brown GC, Felberg NT, Fenton SM: Experimental ocular angiogenesis. Am J Ophthalmol 1980, 89: $231-7$.

7. Knox DL: Ischaemic ocular inflammation. Am J Ophthalmol 1965, 60: 995-1002.

8. Sturrock GD and Mueller HR: Chronic ocular ischaemia. $\mathrm{Br}$ J Ophthalmol 1984, 68: 716-23.

9. Willerson D and Aaberg TM: Acute central retinal artery occlusion and optic disc neovascularisation: occurrence in a patient with carotid artery disease. Arch Ophthalmol 1978, 96: 451-2.

10. Aapen RE, Wray SA, Cogan DG: Central retinal artery occlusion. Am J Ophthalmol 1975, 79: 374-81.

11. Kearns TP and Hollenhorst RW: Venous stasis retinopathy of occlusive disease of the carotid artery. Mayo Clin Proc 1963, 38: 304-12.

12. Kearns TP, Siebert RG, Sundt TM: The ocular aspects of by-pass surgery of the carotid artery. Mayo Clin Proc 1979; 54: $3-11$.

13. Perraut LE and Zimmerman LE: The occurrence of glaucoma following occlusion of the central retinal artery. Arch Ophthalmol 1959, 61: 845-65.

14. Wolter JR and Phillips RL: Secondary glaucoma following occlusion of the central retinal artery. Am J Ophthalmol 1959, 47: 335-40.

15. Wolter JR: Double embolism of the central retinal artery followed by secondary haemorrhagic glaucoma. Am J Ophthalmol 1972, 73: 651-7.

16. Wolter JR and Phillips RL: Secondary glaucoma in cranial arteritis. Am J Ophthalmol 1965, 59: 625-34.

17. Kottow $M$ and Hendrickson $P$ : Iris angiographic findings in retinal arterial occlusions. Can J Ophthalmol 1974, 9: $435-43$.

18. Brown GC: Isolated central retinal artery obstruction in association with ocular neovascularisation. Am J Ophthalmol 1983, 96: 110-11. 